\title{
Tight junctions and compression therapy in chronic venous insufficiency
}

\author{
YARED HEROUY ${ }^{1,4}$, BIRGIT KAHLE $^{2}$, MARCO IDZKO $^{3}$, INGO EBERTH $^{4}$, JOHANNES NORGAUER $^{5}$, \\ FELIZITAS PANNIER $^{6}$, EBERHARD RABE ${ }^{6}$, MICHAEL JÜNGER ${ }^{7}$ and LEENA BRUCKNER-TUDERMAN ${ }^{4}$ \\ ${ }^{1}$ Dermato-Phlebologische Praxis, D-76530 Baden-Baden; ${ }^{2}$ Department of Dermatology, University Hospital, D-23538 \\ Lübeck; ${ }^{3}$ Department of Pneumology, University Hospital, D-79102 Freiburg; ${ }^{4}$ Department of Dermatology, University \\ Hospital, D-79104 Freiburg; ${ }^{5}$ Department of Dermatology, University Hospital, D-07740 Jena; ${ }^{6}$ Department of Dermatology, \\ University Hospital, D-53105 Bonn; ${ }^{7}$ Department of Dermatology, University Hospital, D-17489 Greifswald, Germany
}

Received January 30, 2006; Accepted March 22, 2006

\begin{abstract}
Tight junctions (TJs) provide a barrier function, inhibiting solute and water flow through the paracellular space. There had been no analysis until now as to how tight junction molecules could be involved in the pathology of patients with chronic venous insufficiency. The aim of the study was to analyse the expression pattern of TJ-molecules occludin (OCLN), claudin-1 (CLDN-1), claudin-3 (CLDN-3) and claudin-5 (CLDN-5) on mRNA and protein level in patients with edema, venous leg ulcers and healthy controls. Biopsy specimens were taken in healthy individuals and in patients before, and four weeks after compression therapy. mRNA-expression was determined by using reversetranscriptase and polymerase chain reaction (RT-PCR) and the protein-expression was determined by Western blotting from tissue specimens. Quantification performed determining the expression for TJ-molecules displayed diminished expression for CLDN-1 $(\mathrm{p}<0.01)$ and CLDN-5 $(\mathrm{p}<0.01)$ in patients with chronic venous insufficiency in comparison with healthy controls on mRNA as well as protein level. No statistical differences were detected for OCLN and CLDN-3 between the edema group and healthy controls. There was a significantly elevated expression $(\mathrm{p}<0.01)$ on mRNA and protein level between the leg ulcer group and healthy controls for OCLN and CLDN-3. Densitometric evaluation revealed a more significantly elevated expression $(\mathrm{p}<0.01)$ for CLDN-1 and CLDN-5 on mRNA and protein level after four weeks of compression therapy in comparison with prior to treatment for the edema as well as the leg ulcer group. Compression therapy tightens the paracellular barrier via elevated expression of specific TJs and prevents thereby the
\end{abstract}

Correspondence to: Dr Yared Herouy, Dermato-Phlebologische Praxis, Sophienstrasse 7, D-76530 Baden-Baden, Germany E-mail: info@herouy.de

Key words: tight junctions, compression therapy, varicose veins, venous insufficiency, leg ulcers progression of chronic venous insufficiency due to inhibited permeability of fluid into the perivascular tissue.

\section{Introduction}

The integrity of interendothelial cell contacts is vital for the physiological role of the endothelium as the interface between the blood and tissue structures. The control of vascular permeability is dependent on the opening and closure or the dissociation and formation of interendothelial cell junctions $(1,2)$. Paracellular permeability across endothelial or epithelial cell layers is determined by tight junctions (TJs) that seal the intercellular space (3). Tight junctions provide a barrier function, inhibiting solute and water flow through the paracellular space $(4,5)$. They are thought to be directly involved in barrier and fence functions by sealing them to generate the primary barrier against the diffusion of solutes through the paracellular pathway and by acting as a boundary between the apical and basolateral plasma membrane domains to create and maintain cell polarity, respectively. There is accumulating evidence that occludin (OCLN) and claudins (CLDNs) constitute the backbone and create the variable properties of tight-junction strands (6). They belong to a family of transmembrane proteins which are proteins with cytoplasmatic amino and carboxyl termini and two extracellular domains (Fig. 1). Most cell types express more than two CLDN species in various combinations to constitute TJ strands. Within individual single strands, distinct species of CLDNs are polymerized together to form 'heteropolymers' and, between adjacent strands within 'paired' strands, CLDNs adhere with each other in a homotypic as well as a heterotypic manner $(7,8)$. The extracellular domains of CLDNs share regions of sequence identity but are quite variable in the position and number of amino acids with charged side chains, suggesting they might have additionally different effects on the paracellular diffusion. However, it seems that the complexity of the TJ strand network and the tightness of TJs is determined by the combination of the different CLDNs (9).

Patients with chronic venous insufficiency early develop edema of the lower extremity (10). Elevated hydrostatic pressure had been thought to be the underlying mechanism of 
A

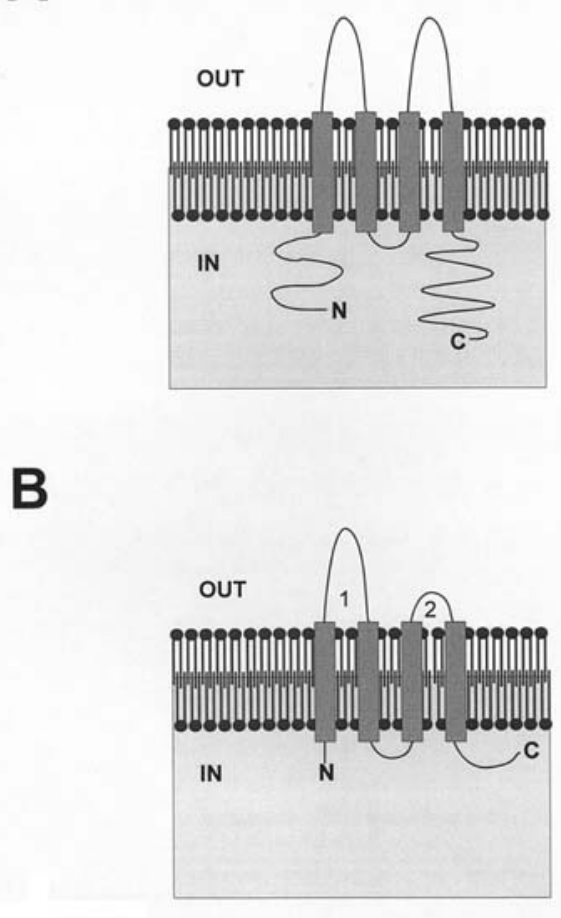

Figure 1. Integral membrane proteins of TJ-molecules. (A) Occludin has four transmembrane domains with two extracellular loops. (B) Claudin-1 is constituted by four transmembrane domains, but shows sequence similarity to OCLN. The cytoplasmatic tail of CLDN-1 is shorter than that of occludin.

elevated leakage of fluid from insufficient vessels into the perivascular tissue causing intradermal edema (11). The lasting presence of intradermal edema may predispose to the development of severe stages of chronic venous insufficiency. There had been no analysis as to how TJ-molecules could be involved in the progression of chronic venous insufficiency. Medical compression stockings are a cornerstone of treatment in these patients since compression garments, when efficiently applied, prevent stasis and support the resorption of intradermal fluid (12). Therefore, we were prompted to question whether compression therapy with compression stockings influences the pattern of TJ-molecule expression in patients with chronic venous insufficiency.

\section{Patients and methods}

Clinical specimens. Patients with chronic venous insufficiencyassociated edema and chronic venous ulcers have been characterized according to the international clinical CEAPclassification of chronic venous insufficiency (CVI). The diagnosis was confirmed clinically by duplex scanning, doppler sonography, and by photoplethysmography. Patients with varicosis-associated edema $(n=8)$ and venous leg ulcers $(n=8)$ received compression stockings (Bauerfeind Phlebologie $\mathrm{GmbH} \& \mathrm{Co} \mathrm{KG}$, Zeulenroda, Germany) over a period of 4 weeks. Punch biopsies were obtained from patients with edema, venous leg ulcers and healthy skin extending to the subcutaneous fat of the lower portion of the leg. In patients with edema and in healthy controls, punch biopsies were taken $2 \mathrm{~cm}$ above the medial malleolus. In patients with venous leg ulcers, specimens were obtained from the ulcer ground. Tissue specimens were taken in patients with chronic venous insufficiency prior to treatment and 4 weeks after treatment with compression stockings. The second tissue specimens were taken $1 \mathrm{~cm}$ from the first biopsy. Patients with venous leg ulcers received Venotrain ulcertec and patients with CVI-associated edema received Venotrain Micro or Venotrain Champion. Biopsy specimens were snap-frozen in liquid nitrogen and stored at $-70^{\circ} \mathrm{C}$ until further processing. Control biopsies from healthy volunteers $(n=8)$ were taken from the lower portion of the leg. All subjects provided informed written consent, and procedures involving humans were approved by the Ethics Committee of the University of Freiburg (Votum-NR.21/2002).

Table I. Designed primers for detecting mRNA expression with RT-PCR.

\begin{tabular}{lll}
\hline OCLN and CLDNs & Sequence (5'-3') & PCR size \\
\hline $\begin{array}{l}\text { OCLN } \\
\text { Sense }\end{array}$ & TCT GCA ACG TTG TCA GAA GG & 204 bp \\
Antisense & ACT CAG TCC TTT AGC ACA ACA GC \\
CLDN-1 & & \\
$\quad$ Sense & TCA GCA CTG CCC TGC CCC AGT & 506 bp \\
Antisense & TGG TGT TGG GTA AGA GGT TGT \\
CLDN-3 & \\
Sense & AAG GCC AAG ATC ACC ATC GTG \\
Antisense & AGA CGT AGT CCT TGC GGT CGT \\
CLDN-5 & \\
Sense & ATG TCG TGC GTG GTG CAG AG \\
Antisense & GGT GCA GAC CCA GGC GCC GCA & 304 bp \\
\hline
\end{tabular}




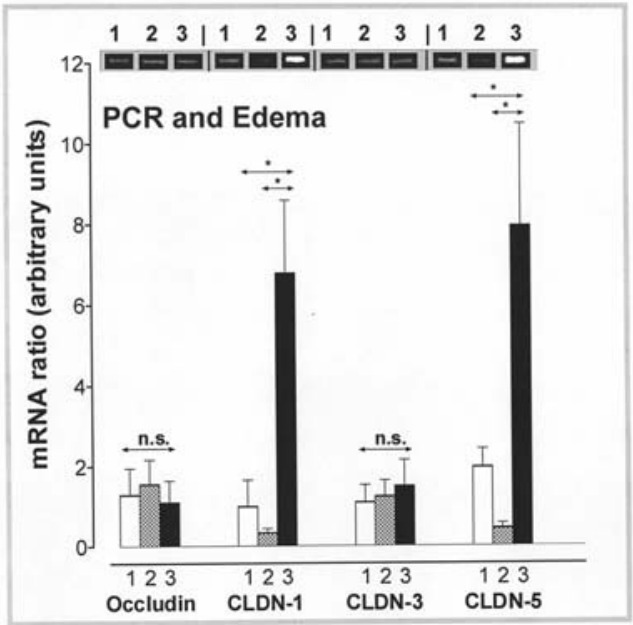

Figure 2. The mRNA expression of OCLN, CLDN-1, CLDN-3 and CLDN-5 in healthy skin (left bands), in patients with edema prior to treatment (middle bands) and after treatment with compression stockings (right bands). (A) Total mRNA was prepared for reverse transcription and amplification of generated cDNA with polymerase chain reaction. Amplified products of OCLN, CLDN-1, CLDN-3 and CLDN-5 and the control gene, GAPDH, were stained by ethidium bromide. Representative data from one patient with edema and one control are shown. (B) Densitometric evaluation of generated mRNAproducts of OCLN, CLDN-1, CLDN-3 and CLDN-5 in healthy controls [(1) white bars], edema patients prior to treatment [(2) crossed bar] and after treatment [(3) black bars] To compare mRNA expression of patients, the obtained signals were normalized to the level of the control gene, GAPDH, by calculating the ratio between the analysed genes and GAPDH. Data are means \pm SEM ( $n=8$ for healthy skin; $n=8$ for edema group). The significance of difference was determined by an unpaired Student's t-test. Differences were considered significant at ${ }^{*} \mathrm{p}<0.01$

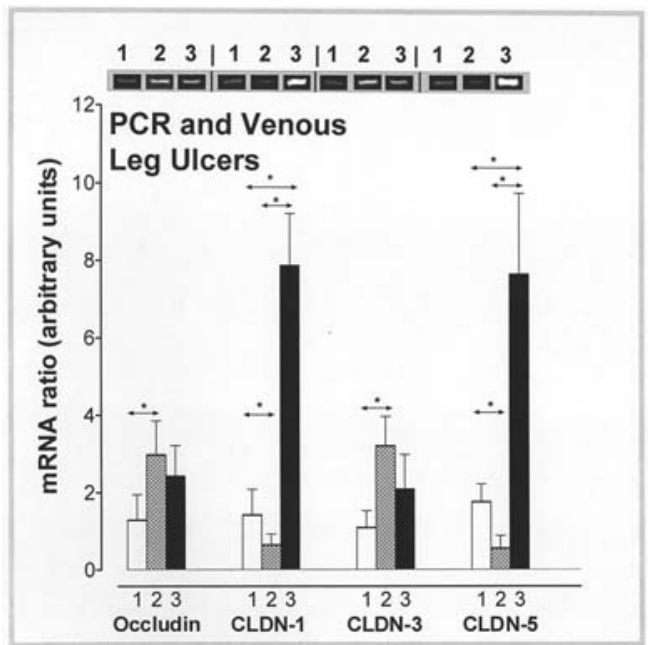

Figure 3. The mRNA expression of OCLN, CLDN-1, CLDN-3 and CLDN-5 in healthy skin (left bands), in patients with venous leg ulcers prior to treatment (middle bands) and after treatment with compression stockings (right bands). (A) Total mRNA was prepared for reverse transcription and amplification of generated cDNA with polymerase chain reaction. Amplified products of OCLN, CLDN-1, CLDN-3 and CLDN-5 and the control gene, GAPDH, were stained by ethidium bromide. Representative data from one patient with venous leg ulcers and one control are shown. (B) Densitometric evaluation of generated mRNA-products of OCLN, CLDN-1, CLDN-3 and CLDN-5 in healthy controls [(1) white bars], patients with venous leg ulcers prior to treatment [(2) crossed bar] and after treatment [(3) black bars]. To compare the mRNA expression of patients, the obtained signals were normalized to the level of the control gene, GAPDH, by calculating the ratio between the analysed genes and GAPDH. Data are means \pm SEM ( $n=8$ for healthy skin; $n=8$ for venous leg ulcer group). The significance of difference was determined by an unpaired Student's t-test. Differences were considered significant at $* \mathrm{p}<0.01$.

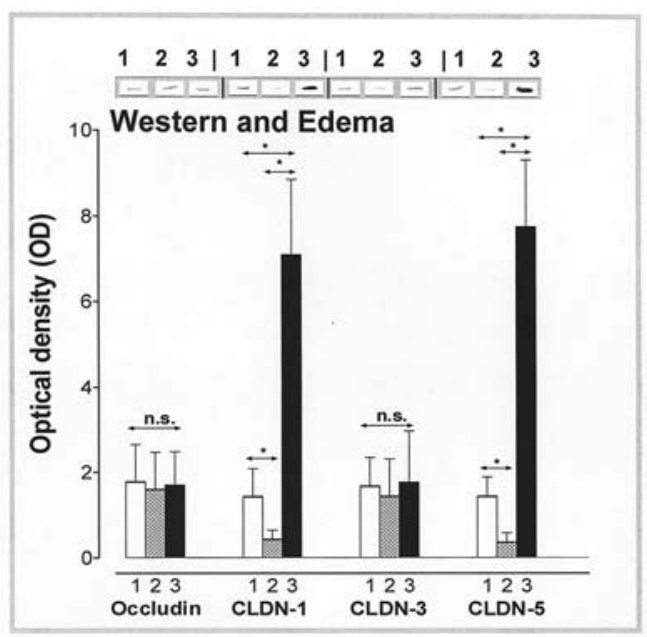

Figure 4. Protein expression of OCLN, CLDN-1, CLDN-3 and CLDN-5 in healthy skin and in patients with edema prior to treatment and after treatment with compression stockings. (A) Western blotting was performed with antibodies against OCLN, CLDN-1, CLDN-3 and CLDN-5 in healthy skin (left blots) and in patients with edema prior (middle blots) and after compression therapy (right blots). (B) Densitometric evaluation of immunoblots of OCLN, CLDN-1, CLDN-3 and CLDN-5 in healthy controls [(1) white bars], edema patients prior to treatment [(2) crossed bar] and after treatment [(3) black bars]. Data are means \pm SEM ( $n=8$ for healthy skin; $n=8$ for edema group). The significance of difference was determined by an unpaired Student's t-test. Differences were considered significant at ${ }^{*} \mathrm{p}<0.01$.

Antibodies. Antibodies against OCLN (mono-Ab. OC-3F10), CLDN-1 (poly-Ab. JAY.8), CLDN-3 (poly-Ab. Z23.JM), and CLDN-5 (Ab. Z43.JK) were purchased from Zymed Laboratories (San Francisco, CA, USA).

Reverse-transcriptase polymerase chain reaction ( $R T-P C R)$. Detection of mRNAs for specific claudins was performed semi-quantitatively by reverse transcriptase and polymerase chain reaction (RT-PCR) (13). Briefly, total mRNA was isolated and cDNA was obtained using the first strand cDNA synthesis kit using mRNA. All oligonucleotides were designed to recognize a unique sequence exclusive for each cDNA for OCLN, CLDN-1, CLDN-3 and CLDN-5 (Tab. I). The control gene, glyceraldehyde-3-phosphate dehydrogenase (GAPDH), served as a reference. Polymerase chain reaction was performed using a GeneAmp PCR thermal cycler (Perkin-Elmer, Weiterstadt, Germany) and consisted of cycles of denaturation $\left(94^{\circ} \mathrm{C}\right.$ for $\left.1 \mathrm{~min}\right)$, ramped annealing $\left(60^{\circ} \mathrm{C}\right.$ for $\left.30 \mathrm{sec}\right)$ and extension $\left(72^{\circ} \mathrm{C}\right.$ for $\left.1 \mathrm{~min}\right)$. The generated products were subjected to electrophoresis on a $3 \%$ agarose gel and were visualized by ethidium bromide staining. To compare the mRNA expression of different biopsies, signals of OCLN, CLDN-1, CLDN-3 and CLDN-5 were normalized to GAPDH and a ratio was calculated. In order to assure linear cDNA amplification in our experiments, different amplifying cycles (22-36) were checked. Linear amplification was obtained between 22 and 32 cycles. The specificity of the generated products was proven by sequencing after cloning using pCRTMII vectors.

Immunoblot analysis. Protein analysis of OCLN, CLDN-1, CLDN-3 and CLDN-5 was performed by Western blotting. Lesion and skin samples were pulverized with a microdismembrator from Braun Biotech International (Melsungen, 


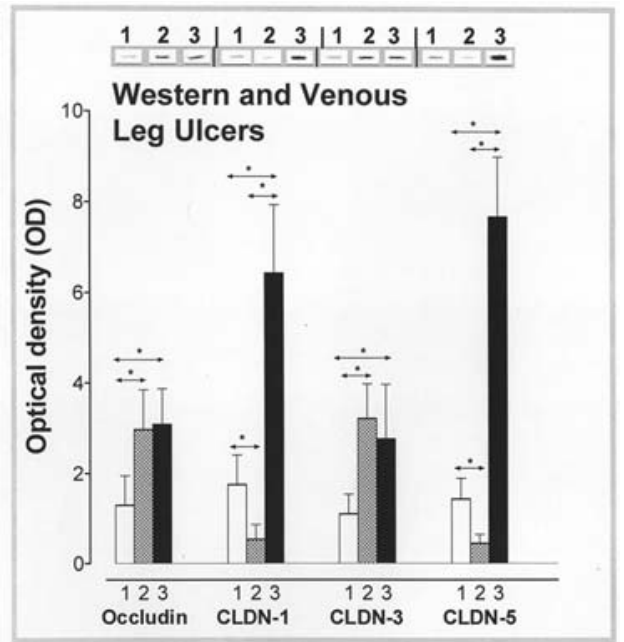

Figure 5. Protein expression of OCLN, CLDN-1, CLDN-3 and CLDN-5 in healthy skin and in patients with venous leg ulcer prior to treatment and after treatment with compression stockings. (A) Western blotting was performed with antibodies against OCLN, CLDN-1, CLDN-3 and CLDN-5 in healthy skin (left blots) and in patients with venous leg ulcers prior (middle blots) and after compression therapy (right blots). (B) Densitometric evaluation of immunoblots of OCLN, CLDN-1, CLDN-3 and CLDN-5 in healthy controls [(1) white bars], patients with venous leg ulcers prior to treatment [(2) crossed bar] and after treatment [(3) black bars]. Data are means \pm SEM ( $n=8$ for healthy skin; $n=8$ for venous leg ulcers group). The significance of difference was determined by an unpaired Student's t-test. Differences were considered significant at ${ }^{*} \mathrm{p}<0.01$

Germany). Proteins were solubilized in electrophoresis sample buffer and samples with equal protein content were separated by $10 \%$ SDS-PAGE. Proteins were electroblotted to nitrocellulose membrane and were stained with indicated antibodies using peroxidase-conjugated secondary antibodies and the ECL chemiluminescence system from Amersham (Braunschweig, Germany).

Densitometric evaluation and statistical analysis. The intensity of bands and blots were quantified measuring the optical density with OneDscan computer software package. Data were analysed by an unpaired Student's t-test. Differences were considered significant at $\mathrm{p}<0.01$. All values were expressed as means \pm SEM.

\section{Results}

Gene expression. Patients with chronic venous insufficiency and healthy controls displayed gene expression for OCLN, CLDN-1, CLDN-3 and CLDN-5. The mRNA expression of OCLN, CLDN-1, CLDN-3 and CLDN-5 in biopsies of edema (Fig. 2), venous leg ulceration (Fig. 3) and healthy controls was analysed semi-quantitatively by RT-PCR. The mRNA-products for TJ-molecules displayed diminished band intensity for CLDN-1 $(\mathrm{p}<0.01)$ and CLDN-5 $(\mathrm{p}<0.01)$ in patients with chronic venous insufficiency in comparison with healthy controls. No difference was detected for OCLN and CLDN-3 with regard to the band intensity between the edema group and healthy controls. However, there was a significantly elevated expression $(\mathrm{p}<0.01)$ on mRNA level between the leg ulcer group and healthy controls for OCLN and CLDN-3 (Fig. 3). Densitometric evaluation revealed a more significantly elevated expression $(\mathrm{p}<0.01)$ for CLDN-1 and CLDN-5 on mRNA level after four weeks of compression therapy in comparison with prior to treatment for the edema (Fig. 2) as well as the leg ulcer group (Fig. 3). No statistical differences could be found for OCLN and CLDN-3 solely for the edema group prior to treatment in comparison with compression therapy treated patients on mRNA-level (Fig. 2). The quantitation performed by determination of the relative expression normalized to the expression of GAPDH demonstrated significant differences.

Protein expression. Patients with chronic venous insufficiency and healthy controls displayed immunoreactivity on protein level for OCLN, CLDN-1, CLDN-3 and CLDN-5. The protein expression of OCLN, CLDN-1, CLDN-3 and CLDN-5 in biopsies of edema (Fig. 4) and venous leg ulceration (Fig. 5) and healthy controls was performed by Western blotting. The immunoreactive blots for TJ-molecules displayed diminished blot intensity for CLDN-1 $(\mathrm{p}<0.01)$ and CLDN-5 $(\mathrm{p}<0.01)$ in patients with chronic venous insufficiency in comparison with healthy controls (Figs. 4 and 5). No difference could be detected for OCLN and CLDN-3 with regard to the blot intensity between the edema group and healthy controls. However, there was a significantly elevated expression $(\mathrm{p}<0.01)$ on protein level between the leg ulcer group and healthy controls for OCLN and CLDN-3 (Fig. 5). Densitometric evaluation revealed a more significantly elevated expression $(\mathrm{p}<0.01)$ for $\mathrm{CLDN}-1$ and CLDN-5 on protein level after four weeks of compression therapy in comparison with prior to treatment for the edema (Fig. 4) as well as the leg ulcer group (Fig. 5). No statistical differences were found for OCLN and CLDN-3 solely for the edema group prior to treatment in comparison with compression therapy treated patients on protein-level (Fig. 4).

\section{Discussion}

Exchange of solutes and ions between the luminal and abluminal compartments of the circulation is critically dependent on the barrier properties of the vascular endothelium. TJs provide a barrier function, inhibiting solute and water flow through the paracellular space. There is accumulating evidence that claudins constitute the backbone of TJ strands and create the variable properties of the barrier $(4,5)$. It had been reported that overexpression of specific claudin molecules in MDCK cell monolayers increased transepithelial electric resistence (6). Clinically, patients with chronic venous insufficiency, early develop edema of the lower extremity, indicating that elevated paracellular permeability could play a crucial role. In patients with severe chronic venous insufficiency, elevated transcapillary and interstitial diffusion of could be found in capillary vessels by using Na-fluorescein (14). The underlying pathogenic mechanism of chronic venous insufficiency have long been a matter of debate. Pressure damaged capillary vessels with leakage of fibrinogen or release of toxic metabolites and elevated expression of adhesion molecules by accumulated leukocytes or cytokine mediated fibrin cuff formation have been suggested (15-19). Impairment of gas and nutrients exchange between blood and dermis has been supposed to be a common feature. There had 
been no analysis as to how TJ-molecules could play a role in the progression of the disease. Furthermore, there were no indications as to how compression therapy could alter the expression of specific TJ-molecules. Our results provide evidence that specific TJ-molecules are downregulated in patients with chronic venous insufficiency in comparison with healthy controls. CLDN-1 and CLDN-5 displayed diminished expression on mRNA- and protein-level in patients with edema and venous leg ulceration in comparison with healthy controls. Compression therapy over four weeks induced the expression of CLDN-1 and CLDN-5. Since the complexity of the TJ strand network and the tightness of TJs are determined by the combination of the different TJmolecules, it is tempting to speculate that elevated paracellular fluid transfer could be based on an altered expression of specific TJ-molecules, such as CLDN-1 and CLDN-5, which differs significantly from the expression pattern in healthy controls. Elevated paracellular fluid transport, due to altered tightness of the paracellular barrier, could possibly additionally explain the clinical observation of edema in patients with chronic venous insufficiency. Whether downregulation of TJmolecules can be directly associated with elevated hydrostatic pressure in varicose veins remains, however, unclear and requires further investigation. Compression therapy induces strongly the expression of CLDN-1 and CLDN-5 in patients with chronic venous insufficiency, which significantly differs from the expression of healthy controls as well as patients prior to treatment. Until now, compression stockings were known to reduce the diameter of veins, thereby increasing flow velocity, to reduce filtration of fluid out of the intravascular space and to improve lymphatic flow, thereby reducing edema. Graduated compression reduces reflux and improves venous outflow, thus decreasing venous pressure at rest and with ambulation. It seems that compression therapy additionally tightens the paracellular barrier by upregulation of specific TJ-molecules such as CLDN-1 and CLDN-5. These results indicate that compression therapy strengthens the veins to prevent leakage of fluid out of the vessels into the tissue space and may prevent the progression of chronic venous insufficiency. In summary, compression therapy isn't only an external mechanical aid to combat varicose vein disease but also provides a vasoprotective role through tightening the paracellular barrier.

\section{Acknowledgements}

The study design on TJs was awarded by the International Union of Phlebology (IUP) with the Bauerfeind Scientific Phlebology Award (BSP-Award) at the 14th World Congress of the International Union of Phlebology (IUP), Rome 2001.

\section{References}

1. Lampugnani MG and Dejana E: Interendothelial junctions: structure, signalling and functional roles. Curr Opin Cell Biol 9: 674-682, 1977

2. Bazzoni $G$ and Dejana E: Pores in the sieve and channels in the wall: control of paracellular permeability by junctional proteins in endothelial cells. Microcirculation 8: 143-152, 2001

3. Tsukita S, Furuse M and Itoh M: Multifunctional strands in tight junctions. Nat Rev Mol Cell Biol 2: 285-293, 2001.

4. Tsukita $S$ and Furuse M: Occludin and claudins in tight-junction strands: leading or supporting players? Trends Cell Biol 9: 268-273, 1999.

5. Sonoda N, Furuse M, Sasaki H, Yonemura S, Katahira J, Horiguchi Y and Tsukita S: Clostridium perfringens enterotoxin fragment removes specific claudins from tight junction strands: Evidence for direct involvement of claudins in tight junction barrier. J Cell Biol 147: 195-204, 1999.

6. McCarthy KM, Francis SA, McCormack JM, Lai J, Rogers RA, Skare IB, Lynch RD and Schneeberger EE: Inducible expression of claudin-1-myc but not occludin-VSV-G results in aberrant tight junction strand formation in MDCK cells. J Cell Sci 19: 3387-3398, 2000.

7. Furuse M, Sasaki H and Tsukita S: Manner of interaction of heterogeneous claudin species within and between tight junction strands. J Cell Biol 147: 891-903, 1999.

8. Tsukita S, Furuse M: Pores in the wall: claudins constitute tight junction strands containing aqueous pores. J Cell Biol 49: 13-16, 2000.

9. Gow A, Southwood CM, Li JS, Pariali M, Riordan GP, Brodie SE, Danias J, Bronstein JM, Kachar B and Lazzarini RA: CNS myelin and sertoli cell tight junction strands are absent in Osp/claudin-11 null mice. Cell 99: 649-659, 1999.

10. Valencia IC, Falabella A, Kirsner RS and Eaglstein WH: Chronic venous insufficiency and venous leg ulceration. J Am Acad Dermatol 44: 401-421, 2001.

11. Chant A: Tissue pressure, posture, and venous ulceration. Lancet 336: 1050-1051, 1990.

12. Motykie GD, Caprini JA, Arcelus JI, Reyna JJ, Overom E and Mokhtee D: Evaluation of therapeutic compression stockings in the treatment of chronic venous insufficiency. Dermatol Surg 25: 116-120, 1999.

13. Herouy Y, May AE, Pornschlegel G, Stetter C, Grenz H, Preissner KT, Schopf E, Norgauer J and Vanscheidt W: Lipodermatosclerosis is characterized by elevated expression and activation of matrix metalloproteinases: implications for venous ulcer formation. J Invest Dermatol 111: 822-827, 1998.

14. Jünger M, Hahn M, Klyscz T and Steins A: Role of microangiopathy in the development of venous leg ulcers. Prog Appl Microcirc 23: 180-190, 1999.

15. Browse NL and Burnand KG: The cause of venous ulceration. Lancet ii: 243-245, 1982.

16. Hahn J, Jünger M, Friedrich B, Zuder D, Steins A, Hahn M and Klyscz T: Cutaneous inflammation limited to the region of the ulcer in chronic venous insufficiency. Vasa 26: 277-281, 1997.

17. Weyl A, Vanscheidt W, Weiss JM, Peschen M, Schopf E and Simon J: Expression of the adhesion molecules ICAM-1, VCAM-1, and E-selectin and their ligands VLA-4 and LFA-1 in chronic venous leg ulcers. J Am Acad Dermatol 34: 418-423, 1996.

18. Coleridge Smith PD, Thomas P, Acurr J and Dormandy JA: Causes of venous ulceration: A new hypothesis. Br Med J 296: 1726-1727, 1988

19. Herrick SE, Sloan P, McGurk M, Freak L, McCollum CN and Ferguson MWJ: Sequential changes in histologic pattern and extracellular matrix deposition during the healing of chronic venous ulcers. Am J Pathol 141: 1085-1095, 1992. 